\title{
Differences in Dementia Care Between Swedish-Born and Foreign-Born from Countries with Different Country Level Socioeconomic Position: A Nationwide Register-Based Study
}

\author{
Emma Lindgren ${ }^{\mathrm{a}}$, Josephine Sörenson ${ }^{\mathrm{a}}$, Carina Wattmo ${ }^{\mathrm{b}}$, Ingemar Kåreholt ${ }^{\mathrm{c}, \mathrm{d}}$ \\ and Katarina Näggab,e,* \\ ${ }^{a}$ Memory Clinic, Skåne University Hospital, Malmö, Sweden \\ ${ }^{\mathrm{b}}$ Clinical Memory Research Unit, Department of Clinical Sciences Malmö, Lund University, Malmö, Sweden \\ ${ }^{\mathrm{c}}$ Aging Research Center (ARC), Karolinska Institutet and Stockholm University, Stockholm, Sweden \\ ${ }^{\mathrm{d}}$ Institute of Gerontology, School of Health and Welfare, Aging Research Network - Jönköping (ARN-J), \\ Jönköping University, Jönköping, Sweden \\ ${ }^{\mathrm{e}}$ Department of Acute Internal Medicine and Geriatrics, Linköping University, Linköping, Sweden
}

Pre-press 10 October 2021

\begin{abstract}
.
Background: With a growing elderly population worldwide, the prevalence of dementia is rapidly increasing. Studies from high income countries have shown that belonging to a minority ethnic group increases the risk of health disadvantages.

Objective: The aim of the present registry-based study was to identify potential differences in diagnostics, treatment, and care of individuals with dementia focusing on foreign-born in Sweden and the impact of country level socioeconomic position (SEP).

Methods: The study was based on a large dataset from the Swedish Dementia Registry (SveDem) and the Swedish Tax Agency's population registry. Data on demographic variables, cognitive tests, clinical assessments, medication, diagnosis, and interventions initiated at diagnosis were collected. Country level SEP was determined by country of birth as classified by World Bank Country and Lending groups.

Results: Of 57,982 patients with dementia registered in SveDem, 7,171 (12.4\%) were foreign-born. The foreign-born were significantly younger at diagnosis $(p<0.001)$, had a lower MMSE score $(p<0.001)$, lower odds of receiving a specific dementia diagnosis $(p<0.001)$, lower use of acetylcholinesterase inhibitors $(p<0.001)$, and overall a higher use of neuroleptics compared with the Swedish-born group. The lower SEP, the greater differences to Swedish-born were seen in many of the examined variables.

Conclusion: There were significant differences in dementia diagnostics, treatment, and care between foreign-born and Swedish-born, a lower SEP indicating greater differences. Further research should focus on various socioeconomic aspects and health care outcomes for a more profound analysis of equity in dementia care.
\end{abstract}

Keywords: Dementia care, differences, immigrant, minority ethnic group, qualitative registry, socioeconomic position

\footnotetext{
*Correspondence to: Katarina Nägga, MD, PhD, Clinical Memory Research Unit, Department of Clinical Sciences Malmö, Lund University, Skånes universitetssjukhus, VE minnessjukdomar, SE-
}

20502 Malmö, Sweden. Tel.: +46 702566 181; E-mail: katarina. nagga@med.lu.se. 


\section{INTRODUCTION}

Dementia is a growing international public health issue that affects the individual, the family, and society at large [1]. As the world's population is aging, the number of people affected by dementia is increasing. The total number of people with dementia worldwide in 2010 was estimated to be about 35.6 million, and it is expected to double nearly every 20 years [2]. Both the World Health Organization and Alzheimer's Disease International [1] recognize dementia as a public health issue and advocate for action at international and national levels based on the principles of inclusion, integration, equity, and evidence [1]. As a result of globalization, there are more individuals moving between countries and the number of migrants is increasing worldwide. Previous studies have shown differences in dementia prevalence and utilization of dementia care between majority and minority ethnic groups within a nation [3-7]. A recent study in Europe estimates that more than 476,000 of the immigrants aged 65 years or older living in Europe has dementia [8]. With a higher number of elderly immigrants, the accessibility and quality of dementia care for this group are important when promoting equity in health care. Equal distribution of health care promotes an equal society in which all individuals benefit from good health $[9,10]$. Common risk factors related to inequity in access and utilization of health services include socioeconomic and cultural factors such as education, ethnicity, sex, and demography [1]. A high prevalence of dementia has also been identified in immigrants from both high and low socioeconomic countries [11, 12]. Previous studies have shown that the pathway to dementia care differs between majority and minority ethnic groups; where minority ethnic groups seek help at a later stage of dementia and experience more barriers [5, 6, 13], and where non-European immigrants with cognitive problems consult later than European immigrants [14]. Minority ethnic groups sometimes have less knowledge about dementia and are more likely to not conceptualize dementia as an illness but as a part of normal aging $[5,15]$. Not speaking the official language has also been identified as an important barrier for diagnosis of dementia [16-18]. Studies in Englishspeaking countries have shown that patients with a non-English-speaking background with dementia were more likely to present at a later stage of their disease and had a higher prevalence of diagnosed dementia compared with English-speaking groups $[17,18]$. A recent review also stated that minority ethnic groups had less access to dementia-specific treatment and research trials and were less likely to move to a long-term care facility once diagnosed with dementia [13]. The definition of a minority ethnic group is a group of people who share a common cultural identity which differs in some way to that of the majority ethnic group in a particular country, whereas the term immigrant has no commonly agreed definition and is here only used when referring to studies using this term [3].

The Swedish National Board of Health and Welfare estimates that there are 130,000-150,000 individuals with dementia in Sweden; the yearly incidence is $20,000-25,000$ [19]. In 2020, there were 2.0 million residents registered with another country of birth in Sweden, accounting for $20 \%$ of the population [20]. In 2070, it is expected that nearly one third of the elderly residents in Sweden are born in another country than Sweden [21]. A majority of foreignborn in Sweden are from countries outside of Europe [20, 22].

The aim of the present registry-based study was to compare differences in dementia diagnostics and treatment between foreign-born and Swedish-born and the impact of country level socioeconomic position (SEP).

\section{MATERIALS AND METHODS}

\section{Study design}

The study was based on a large dataset obtained from the Swedish Dementia Registry (SveDem) and the Swedish Tax Agency's population registry. The two datasets were merged based on the Swedish personal identification numbers by a data manager. Data on all persons with a dementia diagnosis registered in SveDem from May 2007 to December 2015 were retrieved and de-identified before delivery to the research group.

SveDem, the national quality registry on dementia disorders, was initiated in May 2007 and covers almost all parts of Sweden [23]. The aim of the registry was to improve the quality of diagnostics, treatment, and care of patients with dementia disorders to provide high-quality care for the whole country. Both specialist (memory clinics) and primary care units can be affiliated to the registry. However, affiliation to the registry is voluntary. In November 2016, the registry included data for more than 62,000 persons with dementia. All memory clinics $(n=60)$ and $76 \%$ of all primary care units $(n=1168)$ in Sweden 
reported data to the registry at that time. The prevalence of older foreign-born ( $\geq 65$ years) in Sweden in 2015 was $12.5 \%$ [24] and 12.1\% in SveDem. Hence, the registry seems to provide adequate coverage of the elderly foreign-born population.

\section{Study population}

A total of 57,982 persons were included in the study. 7,171 (12.4\%) of these were born in other countries than Sweden. Forty-two individuals (mean \pm standard deviation [SD] age, $85.3 \pm 5.7$ years; $54.8 \%$ women) were excluded because their personal identity number could not be identified in the Swedish Tax Agency's registry.

\section{Measures}

The following variables were extracted from the SveDem registry: gender, age, dementia diagnosis, and Mini-Mental State Examination (MMSE) score at dementia diagnosis. In addition, the following components of dementia diagnostics were included (performed: yes/no): blood tests; The clock test; computed tomography (CT) or magnetic resonance imaging (MRI) of the brain; lumbar puncture; singlephoton emission computed tomography (SPECT); electroencephalography (EEG); assessments performed by an occupational therapist, physiotherapist, speech therapist, or neuropsychologist. Data of diagnosis were dichotomized to whether a specific dementia diagnosis was registered or not (yes/no). All dementia diagnoses were categorized as specific except for the unspecified dementia diagnosis that was not. The following data from the time of diagnosis were also included: residency, coresidency, diagnosed at a specialist care or a primary care unit; medication with the following drugs (yes/no): antidementia drugs (acetylcholinesterase inhibitors (AC hEI) and/or N-methyl-D-aspartate (NMDA) receptor antagonist); anti-depressants; anti-psychotics; anxiolytics; hypnotics, analgesics, or cardiovascular drugs. Finally, data on initiation of care arrangements were extracted (performed: yes/no): contact with counsellor; contact with social services, as well as support for informal caregivers.

Data on country of birth were retrieved from the Swedish Tax Agency registry. Swedish residents registered with another country of birth than Sweden were divided into three groups depending on country level SEP decided by country of birth. SEP of each country of birth was determined by classification of country income (using gross national income per capita) according to World Bank Country and Lending Groups [25]. Countries of birth with high income economy was defined as high SEP, countries of birth with upper-middle income economy were defined as middle SEP, and countries of birth with lowermiddle- and low-income economy was defined as low SEP. All patients born in Sweden were grouped together in one category named Swedish-born. For more detailed description of the country income classification, please see Supplementary Table 1.

\section{Statistical analysis}

The characteristics of the Swedish-born group and the three foreign-born groups with dementia were compared using statistical analysis. For group comparisons, analysis of variance (ANOVA) for normally distributed continuous variables and chi-squared tests for categorical data were used. ANOVA was used to investigate differences in age and MMSE scores between the four groups.

The association between country specific SEP for the country of birth as main independent variable and variables related to dementia care as dependent variables was analyzed by binomial logistic regression with the Swedish-born group as reference. The analyses were adjusted for age at diagnosis, sex, residency, coresidency, and place of diagnosis (specialist unit or not). The results are presented as odds ratios (OR) and $p$-values.

Statistical analyses were performed using the SPSS statistical package for the Social Sciences [26].

\section{Ethics}

The study was approved by the regional Ethics Review Board in Lund, Sweden.

\section{RESULTS}

\section{Characteristics of patients registered in SveDem}

The mean age $( \pm$ SD) of the whole study population was $80.1 \pm 7.9$ years (range, $28-104$ years) and $34,233(59 \%)$ were female. Of the foreign-born registered in SveDem, 50\% were born in Nordic countries, $36 \%$ in other European countries (including former Soviet Union, Czechoslovakia and Yugoslavia), and $14 \%$ in other parts of the world. Table 1 shows the baseline characteristics of the study population divided into four groups, as described above. 
Table 1

Demographics, tests, diagnosis or not, drugs, and dementia care among Swedish-born patients and foreign-born patients classified by country level socioeconomic position (SEP) $(n=57,982)$

\begin{tabular}{|c|c|c|c|c|c|}
\hline & Sweden & High SEP & Middle SEP & Low SEP & $p^{*}$ \\
\hline$N$ & 50,811 & 5,541 & 1,333 & 297 & \\
\hline Age, mean (sd) & $80.3(7.9)$ & $79.3(7.6)$ & $77.1(9.0)$ & $73.4(10.2)$ & $<0.001$ \\
\hline Women, \% & 58.6 & 63.5 & 57.4 & 52.5 & $<0.001$ \\
\hline $\begin{array}{l}\text { Residency, nursing homes } \\
\quad(n=57,982)\end{array}$ & 11.0 & 10.7 & 9.6 & 5.4 & 0.007 \\
\hline Specialist unit $(n=57,982)$ & 55.1 & 61.1 & 65.9 & 74.1 & $<0.001$ \\
\hline MMSE score, mean (sd) & $19.6(7.2)$ & $18.1(7.6)$ & $13.6(8.5)$ & $12.9(8.6)$ & $<0.001$ \\
\hline MMSE test $(n=57,847)$ & 93.1 & 91.6 & 79.4 & 79.1 & $<0.001$ \\
\hline Clock test $(n=56,003)$ & 86.2 & 84.7 & 75.9 & 71.1 & $<0.001$ \\
\hline Blood test $(n=56,183)$ & 95.3 & 95.1 & 93.9 & 94.8 & 0.137 \\
\hline CT/MRI $(n=56,267)$ & 88.1 & 91.1 & 90.4 & 92.1 & $<0.001$ \\
\hline $\mathrm{LP}(n=55,639)$ & 26.3 & 28.5 & 26.4 & 38.7 & $<0.001$ \\
\hline SPECT $(n=55,319)$ & 6.9 & 5.2 & 5.8 & 6.4 & $<0.001$ \\
\hline $\mathrm{EEG}(n=55,307)$ & 10.4 & 14.4 & 16.6 & 24.9 & $<0.001$ \\
\hline $\begin{array}{l}\text { Occupational therapist } \\
\quad(n=55,382)\end{array}$ & 39.7 & 45.5 & 41.0 & 46.1 & $<0.001$ \\
\hline Physiotherapist $(n=55,174)$ & 6.8 & 6.4 & 5.0 & 7.4 & 0.074 \\
\hline Speech therapist $(n=55,227)$ & 3.8 & 3.6 & 2.4 & 4.9 & 0.049 \\
\hline Neuropsychologist $(n=55,155)$ & 18.0 & 19.1 & 12.3 & 16.3 & $<0.001$ \\
\hline Diagnosis, specific $(n=57,982)$ & 74.9 & 76.5 & 72.7 & 75.4 & 0.011 \\
\hline Anti-dementia drugs ** $(29,415)$ & 75.1 & 72.9 & 76.3 & 71.9 & 0.051 \\
\hline $\mathrm{AChEI}^{* *}(n=29,743)$ & 63.4 & 59.6 & 57.6 & 54.0 & $<0.001$ \\
\hline $\begin{array}{l}\text { NMDA receptor antagonist } * * \\
\quad(n=29,464)\end{array}$ & 14.0 & 15.3 & 21.7 & 19.4 & $<0.001$ \\
\hline Cardiovascular drugs $(n=55,411)$ & 68.3 & 69.9 & 71.5 & 64.4 & 0.004 \\
\hline Anti-depressants (55.240) & 29.0 & 26.0 & 34.6 & 29.8 & $<0.001$ \\
\hline Anxiolytics $(n=55,068)$ & 11.4 & 10.8 & 13.9 & 9.6 & 0.013 \\
\hline Anti-psychotics $(n=55,063)$ & 6.3 & 8.2 & 10.9 & 7.4 & $<0.001$ \\
\hline Hypnotics $(n=55,008)$ & 17.3 & 18.3 & 20.0 & 14.2 & 0.010 \\
\hline Analgesics $(n=25,813)$ & 20.8 & 21.5 & 27.2 & 22.7 & 0.001 \\
\hline Social service contact $(n=55,037)$ & 52.2 & 54.9 & 56.5 & 67.6 & $<0.001$ \\
\hline Counsellor contact $(n=54,366)$ & 10.7 & 11.1 & 15.1 & 19.9 & $<0.001$ \\
\hline Codependent support $(n=52,689)$ & 59.5 & 56.6 & 61.1 & 60.0 & 0.001 \\
\hline
\end{tabular}

Data is presented as percentages. Classification of foreign-born patient by country level SEP, see Supplementary Table 1. SEP, socioeconomic position; MMSE, Mini-Mental State Examination; LP, lumbar puncture; AChEI, acetylcholinesterase inhibitors; NMDA receptor antagonist, N-methyl-D-aspartate receptor antagonist. * $p$-values based on $\chi^{2}$ except for age and MMSE score based on ANOVA. **Includes Alzheimer's disease dementia, mixed dementia, Lewy Body dementia, and Parkinson's disease dementia.

\section{Characteristics of patients by country of birth and country level socioeconomic position}

Univariate analysis revealed that the mean age at diagnosis significantly differed between the four groups $(p<0.001)$ with a lower mean age at diagnosis in the foreign-born groups compared to the Swedish-born group, decreasing with a lower SEP. The proportion of women differed between the four groups $(p<0.001)$, where both the highest and the lowest proportion of women was seen in the foreignborn groups (63.5\% women in high SEP countries; $52.5 \%$ women in low SEP countries respectively). Further, there were differences in residency between the groups $(p=0.007)$ with a lower proportion nursing home residency in the foreign-born groups compared to the Swedish-born group, decreasing with a lower SEP. The mean MMSE score $( \pm$ SD) at diagnosis was significantly lower in the foreignborn groups compared with the Swedish-born group, decreasing the lower country level SEP $(p<0.001)$ (Table 1). For further univariate differences between the groups of countries regarding diagnostic procedures, medical treatments, and care arrangements at diagnoses, please see Table 1.

\section{Dementia care and support in relation to country of birth}

Being foreign-born differs in care of dementia compared to the Swedish-born. Several of the differences in the univariate analysis remained significant after controlling for age at diagnosis, sex, residency, coresidency, and being diagnosed at a specialist unit 
Table 2

Tests, diagnosis or not, dementia care, and support among different foreign-born patients classified by country level SEP with Sweden as reference category $(n=57,982)$

\begin{tabular}{|c|c|c|c|c|}
\hline & High SEP & Middle SEP & Low SEP & Total $p$ \\
\hline$N$ & 5,541 & 1,333 & 297 & \\
\hline \multicolumn{5}{|l|}{ Dependent variables } \\
\hline Residency, nursing homes & $0.74(<0.001)$ & $0.21(<0.001)$ & $0.18(<0.001)$ & $<0.001$ \\
\hline Specialist unit & $1.22(<0.001)$ & $1.35(<0.001)$ & $1.66(<0.001)$ & $<0.001$ \\
\hline MMSE test & $0.74(<0.001)$ & $0.21(<0.001)$ & $0.18(<0.001)$ & $<0.001$ \\
\hline Clock test & $0.81(<0.001)$ & $0.40(<0.001)$ & $0.26(<0.001)$ & $<0.001$ \\
\hline Blood test & $0.92(0.231)$ & $0.67(0.001)$ & $0.71(0.199)$ & 0.003 \\
\hline CT/MRI & $1.20(0.003)$ & $0.84(0.097)$ & $0.69(0.117)$ & 0.002 \\
\hline LP & $0.96(0.344)$ & $0.57(<0.001)$ & $0.66(0.004)$ & $<0.001$ \\
\hline SPECT & $0.66(<0.001)$ & $0.49(<0.001)$ & $0.33(<0.001)$ & $<0.001$ \\
\hline EEG & $1.32(<0.001)$ & $1.40(<0.001)$ & $1.96(<0.001)$ & $<0.001$ \\
\hline Occupational therapist & $1.22(<0.001)$ & $1.00(0.980)$ & $1.23(0.093)$ & $<0.001$ \\
\hline Physiotherapist & $0.95(0.378)$ & $0.73(0.015)$ & $1.11(0.639)$ & 0.078 \\
\hline Speech therapist & $0.92(0.278)$ & $0.55(0.001)$ & $0.99(0.957)$ & 0.011 \\
\hline Neuropsychologist & $0.96(0.327)$ & $0.41(<0.001)$ & $0.41(<0.001)$ & $<0.001$ \\
\hline Diagnosis, specific & $0.97(0.348)$ & $0.66(<0.001)$ & $0.61(0.001)$ & $<0.001$ \\
\hline Anti-dementia drugs* & $0.83(<0.001)$ & $0.93(0.478)$ & $0.59(0.008)$ & $<0.001$ \\
\hline AChEI* & $0.80(<0.001)$ & $0.69(<0.001)$ & $0.48(<0.001)$ & $<0.001$ \\
\hline NMDA receptor antagonist* & $1.12(0.041)$ & $1.70(<0.001)$ & $1.55(0.043)$ & $<0.001$ \\
\hline Cardiovascular drugs & $1.14(<0.001)$ & $1.30(<0.001)$ & $1.04(0.774)$ & $<0.001$ \\
\hline Anti-depressants & $0.82(<0.001)$ & $1.23(0.001)$ & $0.95(0.677)$ & $<0.001$ \\
\hline Anti-psychotics & $1.29(<0.001)$ & $1.78(<0.001)$ & $1.18(0.479)$ & $<0.001$ \\
\hline Anxiolytics & $0.96(0.368)$ & $1.39(<0.001)$ & $1.05(0.803)$ & 0.001 \\
\hline Hypnotics & $1.09(0.028)$ & $1.30(<0.001)$ & $0.97(0.880)$ & 0.001 \\
\hline Analgesics & $1.12(0.039)$ & $1.80(<0.001)$ & $1.80(0.002)$ & $<0.001$ \\
\hline Social service contact & $1.14(<0.001)$ & $1.40(<0.001)$ & $3.06(<0.001)$ & $<0.001$ \\
\hline Counsellor contact & $0.93(0.144)$ & $1.08(0.388)$ & $1.05(0.747)$ & 0.368 \\
\hline Codependent support & $0.87(<0.001)$ & $1.02(0.794)$ & $0.90(0.414)$ & $<0.001$ \\
\hline
\end{tabular}

Data is presented as OR ( $p$-values) based on logistic regressions; Tests, dementia care, and support as dependent variables and country income as main independent variable. Sweden $(n=50,811)$ as reference. Controlled for age at diagnosis, sex, residency, coresidency, and place of diagnosis (specialist unit or not). Classification of foreign-born patient by country level SEP, see Supplementary Table 1. SEP, socioeconomic position; MMSE, Mini-Mental State Examination; LP, lumbar puncture; AChEI, acetylcholinesterase inhibitors; NMDA receptor antagonist, N-methyl-D-aspartate receptor antagonist. *Includes Alzheimer's disease dementia, mixed dementia, Lewy Body dementia, and Parkinson's disease dementia.

or not. Overall, there were differences between the Swedish-born and the foreign-born groups, with a recurring pattern of greater differences to Sweden the lower SEP, defined by country of birth (Table 2).

Adjusted logistic regression models revealed that the differences in residency seen in Table 1 remained with lower odds of foreign-born for nursing home residency, the odds decreased the lower SEP. The odds for receiving the dementia diagnosis at a specialist unit was significantly higher in the foreign-born groups, the odds increased the lower SEP (Table 2).

There were significant differences in the diagnostic assessments. With a lower country level SEP in the foreign-born groups, the odds were lower to have undergone test of MMSE, clock test, SPECT, and neuropsychological assessment $(p<0.001$, respectively). In contrast, there was higher odds having undergone EEG in the foreign-born groups with the opposite ratio $(p<0.001)$. Moreover, there were lower odds having lumbar puncture $(p<0.001)$ in the foreign-born groups and higher odds of assessment by occupational therapist assessments $(p<0.001)$, compared with the Swedish-born.

It was less likely to receive a specific dementia diagnosis in the foreign-born groups compared with the Swedish-born group $(p<0.001)$. Regarding patients with Alzheimer's dementia (AD), mixed dementia, Lewy Body dementia, and Parkinson's disease dementia there were differences in the use of specific anti-dementia drugs between the groups $(p<0.001)$. The use of AChEIs was lower in the foreign-born groups compared with the Swedishborn group $(p<0.001)$, decreasing the lower country level SEP. Treatment with NMDA receptor antagonists also differed between the groups $(p<0.001)$ and was more frequent in the foreign-born groups. In addition, the use of anti-psychotics $(p<0.001)$ and analgesics $(p<0.001)$ were overall significantly higher in foreign-born groups compared with the Swedish-born group (Table 2). 
There were no significant differences in counsellor contact between the groups. The odds were higher to have a social service contact in the foreign-born groups $(p<0.001)$, compared with the Swedish-born.

\section{DISCUSSION}

The study results show that foreign-born registered in SveDem were significantly younger, had overall a higher female representation, and had a lower MMSE score at the time of diagnosis compared with Swedish-born. Overall, a lower country level SEP in the foreign-born groups was associated to greater differences in dementia treatment and care compared with the Swedish-born group.

In 2010, national guidelines for dementia care were introduced in Sweden [27]. An evaluation of the guidelines [28] revealed some shortcomings in the detection and investigation of dementia for people from countries other than Sweden and in services provided. The present registry-based study provides insight into the differences in dementia diagnostics and treatment between foreign-born and Swedishborn patients with dementia and an impact of SEP.

In line with the current study results, previous studies in Denmark and Norway have shown a similar age discrepancy at dementia diagnosis with immigrants and minority ethnic groups overrepresented at younger ages $[4,6]$. In the UK, minority ethnic groups were significantly younger and had a lower MMSE score than the majority ethnic group after controlling for multiple confounders [29]. A lower socioeconomic status has previously been identified as a possible risk factor for the development of dementia [30, 31] and correspond to findings in this study. Also, a lower MMSE score at diagnosis has been proposed to reflect a higher proportion of misdiagnosis of dementia in immigrant populations [32], which might be one explanation to the abovementioned observations. The lower MMSE score also correlates with previous studies of minority ethnic groups, mostly from the United States, where people belonging to a minority ethnic group access diagnostic services at a later stage of the disease [13]. The variance has been recognized as differences in pathways to care and in perceptions of dementia $[18,33]$ and may be related to lower health literacy among individuals with low social position and in refugee populations [34, 35]. However, the lower MMSE score in the immigrant group may also indicate low validity of the MMSE test, since the test has been criticized as having cultural biases being affected by language and education level [36]. In our data, MMSE was not performed for $21 \%$ of the foreign-born in middle and low SEP group, compared with $8 \%$ of the high SEP group and 7\% of the Swedish-born group. There were also significant differences with regard to simple cognitive tests such as the clock test; this was not performed in $24 \%$ respective $29 \%$ of the foreignborn middle and low SEP group compared with $15 \%$ of foreign-born high SEP group and 14\% of Swedishborn group (Table 1). One plausible explanation for the discrepancy is the difficulty in administering and interpreting the results of cognitive tests in individuals with little or varying knowledge of the Swedish language. Language barriers have previously been found to interfere with dementia diagnosis $[16,18$, 37] at the early stages [18], reinforcing the plausibility of this interpretation. On the other hand, two of the foreign-born groups had higher odds for undergoing functional assessment by an occupational therapist. To evaluate the effects in the daily life of a person's impairments and describe necessary adaptations to maintain independence in activities of daily living, a functional evaluation is often an essential complement to the cognitive test results [38]. In cases where the cognitive tests might be disputed from cultural or language barriers, this complementary assessment might have an even higher value in the diagnostic procedure. A survey conducted in 36 dementia centers in Europe showed that only two-thirds of the included centers perform cognitive testing in minority ethnic patients [39]. Communication problems and a lack of adequate cognitive assessment tools are two important factors that deserve more focus in the future to improve early and adequate dementia diagnosis in the immigrant population.

Even though more subjected to the above-described functional assessment, there was a significantly lower likelihood for a specific dementia diagnoses in the foreign-born groups with middle and low SEP $(<0.001)$. This has also been found in foreign-born in the U.S., where a limited English proficiency was seen as a mediator of having undiagnosed dementia [17].

A meta-analysis from 2010 states that ethnic minority groups are less likely to access dementia medication [13]. Recent studies of dementia and immigrants in Nordic countries have highlighted differences in medical treatment. Studies in Norway and Denmark show that immigrants have a lower likelihood of receiving anti-dementia drug therapy [4, 7]. The present data show a similar pattern whereby 
the foreign-born groups differ from the Swedishborn in prescribed anti-dementia drug treatment. In cases with a specific AD diagnosis, there was a difference in drug use whereby foreign-born patients were prescribed more frequent with memantine and less frequent with AChEIs. At the same time, the foreign-born groups had overall a higher likelihood being prescribed with cardiovascular drugs, indicating a higher prevalence of cardiovascular disorders. This may provide one explanation for the observed differences because AChEIs might have adverse cardiovascular effects [40] and hence memantine was the treatment of choice.

There was a significantly higher likelihood for receiving anti-psychotics overall in the foreign-born groups, with highest likelihood in the middle SEP group. These discrepancies in drug treatment are worrisome. The results correspond with earlier criticism from the National Board of Health and Welfare [28] of high use of neuroleptics in Swedish dementia care. Anti-psychotics are mainly recommended for psychotic symptoms and should be used with caution in dementia disorders to prevent serious adverse events, such as cerebrovascular events and mortality [41, 42]. Differences in stage of disease and comorbidity between the groups could explain the differences in drug treatment. However, we were not able to investigate such influence in this study. Another possible explanation might be differences in cultural biases or language barriers as previously mentioned.

There were also differences observed in nonpharmacological care. The foreign-born groups had higher likelihood for contact with the social services. This may be related to disparities in socioeconomic factors in these groups [32] such as greater need for contact with the social support system. Equity does not mean the same treatment and care for all but rather equity in health outcomes of care and treatment [43]. This is in line with person-centered care as recommended in the Swedish guidelines for dementia care [19]. Person-centered care where the diversity of care users is recognized promotes equity and avoids possible category fallacy in ethnicity $[44,45]$.

There were several differences in the studied variables between the foreign-born groups. Foreign-born from middle- or low SEP countries differed considerably from foreign-born from countries with high SEP, the latter being more similar to Swedish-born. The results in previous studies with minority ethnic groups from other countries than Europe are in line with our findings. In Belgium, non-European immigrants were significantly younger at diagnosis of $\mathrm{AD}$ than Belgium-born patients and had lower MMSE score after controlling for sex, age, and education [14]. For non-western immigrants in the Netherlands, the prevalence of mild cognitive impairment and dementia were three to four times higher than the native Dutch population. However, significant differences in education and socioeconomic status between the groups were not controlled for [12]. With a growing number of foreign-born from countries with middle- or low SEP in Sweden, it is important to acknowledge these vulnerable groups to ensure equal treatment and care for all.

Studies comparing foreign-born groups depending on country level SEP and dementia treatment and care are rare. The strength of the study is the large cohort of patients registered in SveDem from Swedish memory clinics and primary care units located in different geographical areas. A limitation is that misdiagnosis of dementia in minority ethnic groups might occur, and the registry-based data does not include the possibility of addressing this issue.

\section{CONCLUSIONS}

There were significant differences in dementia diagnostics, treatment and care between patients being foreign-born and Swedish-born. The foreignborn groups were younger, had overall a higher female representation and lower MMSE score at the time of diagnosis. They were also treated less frequent with anti-dementia drugs and more often with anti-psychotics. Lower country level SEP in the foreign-born groups was associated to greater differences compared with the Swedish-born patients in many of the examined variables. The result could reflect challenges in communication and higher exposure to modifiable environmental and lifestyle risk factors in the foreign-born groups with middle or low SEP. A person-centered care with culturally appropriate cognitive assessment scales and an awareness of the impact of education and language in dementia assessment promotes an intercultural care which ensure equal treatment of people from all ethnic groups. Further research should focus on socioeconomic factors and health care outcomes for a more profound analysis of equity in dementia care. Public health policies and interventions regarding dementia need to acknowledge foreign-born with middle or low SEP as an exposed group. Moreover, the results of this study imply a need for greater focus on 
persons from minority ethnic groups with young onset dementia.

\section{ACKNOWLEDGMENTS}

The authors thank Spencer Clarkson for help with data management. We also want to express our gratitude to all participants in the study. The study was funded by Migrationsskolan, Memory Clinic, Skåne University Hospital, Malmö, Sweden.

Authors' disclosures available online (https:// www.j-alz.com/manuscript-disclosures/21-0734r1).

\section{SUPPLEMENTARY MATERIAL}

The supplementary material is available in the electronic version of this article: https://dx.doi.org/ 10.3233/JAD-210734.

\section{REFERENCES}

[1] World Health Organization \& Alzheimer's Disease International (2012) Dementia: A Public Health Priority. World Health Organization \& Alzheimer's Disease International, Geneva.

[2] Prince M, Bryce R, Albanese E, Wimo A, Ribeiro W, Ferri CP (2013) The global prevalence of dementia: A systematic review and metaanalysis. Alzheimers Dement 9, 63-75.

[3] Alzheimer Europe (2018) The development of intercultural care and support for people with dementia from minority ethnic groups. Alzheimer Europe.

[4] Diaz E, Kumar BN, Engedal K (2015) Immigrant patients with dementia and memory impairment in primary health care in Norway: A national registry study. Dement Geriatr Cogn Dis 39, 321-331.

[5] Mukadam N, Cooper C, Livingston G (2011) A systematic review of ethnicity and pathways to care in dementia. Int $J$ Geriatr Psychiatry 26, 12-20.

[6] Nielsen TR, Vogel A, Phung TKT, Gade A, Waldemar G (2011) Over- and under-diagnosis of dementia in ethnic minorities: A nationwide register-based study. Int J Geriatr Psychiatry 26, 1128-1135.

[7] Stevnsborg L, Jensen-Dahm C, Nielsen TR, Gasse C, Waldemar G (2016) Inequalities in access to treatment and care for patients with dementia and immigrant background: A Danish nationwide study. J Alzheimers Dis 54, 505-514.

[8] Canevelli, M, Lacorte E, Cova I, Zaccaria V, Valletta M, Raganato R, Bruno G, Bargagli AM, Pomati S, Pantoni L, Vanacore N (2019) Estimating dementia cases amongst migrants living in Europe. Eur J Neurol 26, 1191-1199.

[9] Marmot M (2005) The Status Syndrome: How Social Standing Affects Our Health and Longevity. Holt Paperbacks, New York.

[10] Wilkinson R (2005) The Impact of Inequality: How to Make Sick Societies Healthier. Routledge, London.

[11] Petersen MS, Lophaven SN, Weihe P, Lynge E (2020) High incidence of dementia in Faroese-born female residents in Denmark. Alzheimers Dement (N Y) 6, e12022.
[12] Parlevliet JL, Uysal-Bozkir Ö, Goudsmit M, van Campen JP, Kok RM, Ter Riet G, Schmand B, de Rooij SE (2016) Prevalence of mild cognitive impairment and dementia in older non-western immigrants in the Netherlands: A crosssectional study. Int J Geriatr Psychiatry 31, 1040-1049.

[13] Cooper C, Tandy AR, Balamurali TBS, Livingston G (2010) A systematic review and meta-analysis of ethnic differences in use of dementia treatment, care, and research. Am J Geriatr Psychiatry 18, 193-203.

[14] Segers K, Benoit F, Colson K, Kovac V, Nury D, Vanderspoilden V (2013) Pioneers in migration, pioneering in dementia: First generation immigrants in a European metropolitan memory clinic. Acta Neurol Belg 113 , 435-440.

[15] Antelius E, Kiwi M (2015) Frankly, none of us know what dementia is: Dementia caregiving among Iranian immigrants living in Sweden. Care Manag J 16, 79-94.

[16] Daker-White G, Beattie AM, Gilliard J, Means R (2002) Minority ethnic groups in dementia care: A review of service needs, service provision and models of good practice. Aging Mental Health 6, 101-108.

[17] Franco Y, Choi EY (2020) The relationship between immigrant status and underdiagnosed dementia: The role of limited English Proficiency. J Immigr Minor Health 22, 914-922.

[18] LoGiudice D, Hassett A, Cook R, Flicker L, Ames D (2001) Equity of access to a memory clinic in Melbourne? NonEnglish speaking background attenders are more severely demented and have increased rates of psychiatric disorders. Int J Geriatr Psychiatry 16, 327-334.

[19] National Board of Health and Welfare (2017) National Guidelines for Care in Cases of Dementia - Support for governing and management. The National Board of Health and Welfare, Stockholm.

[20] Statistics Sweden (2021) Foreign-born by Country of birth, sex and year of immigration 31 December 2020. In Population statistics, https://scb.se/en/findingstatistics/statistics-by-subject-area/population/populationcomposition/population-statistics/ Last updated February 22, 2021, Accessed on June 4 January 29, 2021.

[21] Statistics Sweden (2020) The future population of Sweden 2019-2070. Statistics Sweden, Stockholm.

[22] Statistics Sweden (2016) From Finland to Afghanistan Immigration and Emigration Since 1970 for Persons Born in Different Countries. Statistics Sweden, Stockholm.

[23] Religa D, Fereshtehnejad SM, Cermakova P, Edlund AK, Garcia-Ptacek S, Granqvist N, Hallbäck A, Kåwe K, Farahmand B, Kilander L, Mattsion UB, Nägga K, Nordström P, Wijk H, Wimo A. Winblad B, Eriksdotter M (2015) SveDem, the Swedish Dementia Registry - A tool for improving the quality of diagnostics, treatment and care of dementia patients in clinical practice, PLoS One 10, 0116538.

[24] Statistics Sweden (2016) Fortsatt ökning av utrikes födda i Sverige, http://www.scb.se/sv」/Hitta-statistik/Artiklar/For tsatt-okning-av-utrikes-fodda-i-Sverige/ Last updated May 5, 2017, Accessed on June 15, 2017.

[25] The World Bank (2021) World Bank Country and Lending Groups. In Data, https://datahelpdesk.worldbank.org/ knowledgebase/articles/906519-world-bank-country-andlending-groups Accessed on June 4 January 29, 2021.

[26] IBM Corp. Released 2017. IBM SPSS Statistics for Windows, Version 25.0. IBM Corp., Armonk, NY.

[27] National Board of Health and Welfare (2010) National Guidelines for Care in Cases of Dementia. The National Board of Health and Welfare, Stockholm. 
[28] National Board of Health and Welfare (2014) National Evaluation - Care in Cases of Dementia 2014. The National Board of Health and Welfare, Stockholm.

[29] Mukadam N, Lewis G, Mueller C, Werbeloff N, Stewart R, Livingston $G$ (2019) Ethnic differences in cognition and age in people diagnosed with dementia: A study of electronic health records in two large mental healthcare providers. Int $J$ Geriatr Psychiatry 34, 504-510.

[30] Fischer C, Yeung E, Hansen T, Gibbons S, Fornazzari L, Ringer L, Schweizer TA (2009) Impact of socioeconomic status on the prevalence of dementia in an inner city memory disorders clinic. Int Psychogeriatr 21, 1096-1104.

[31] Sattler C, Toro P, Schönknecht P, Schröder J (2012) Cognitive activity, education and socioeconomic status as preventive factors for mild cognitive impairment and Alzheimer's disease. Psychiatr Res 196, 90-95.

[32] Parker C, Philp I (2004) Screening for cognitive impairment among older people in black and minority ethnic groups. Age Ageing 33, 447-452.

[33] Dilworth-Anderson P, Gibson BE (2002) The cultural influence of values, norms, meanings, and perceptions in understanding dementia in ethnic minorities. Alzheimer Dis Assoc Dis 16, 56-63.

[34] Sørensen K, Pelikan JM, Röthlin F, Ganahl K, Slonska Z, Doyle G, Fullam J, Kondilis B, Agrafiotis D, Uiters E, Falcon M, Mensing M, Tchamov K, van den Broucke S, Brand (2015) Health literacy in Europe: Comparative results of the European health literacy survey (HLS-EU). Eur J Public Health 25, 1053-1058.

[35] Wångdahl J, Lytsy P, Mårtensson L, Westling R (2015) Health literacy among refugees' experiences of the health examination for asylum seekers - a Swedish cross-sectional study. BMC Public Health 15, 1162.

[36] Naqvi RM, Haider S, Tomlinson G, Alibhai S (2015) Cognitive assessments in multicultural populations using the Rowland Universal Dementia Assessment Scale: A systematic review and meta-analysis, Can Med Assoc J 187, 169-175.
[37] Sagbakken M, Spilker SR, Nilesen TR (2018) Dementia and immigrant groups: A qualitative study of challenges related to identifying, assessing, and diagnosing dementia. $B M C$ Health Serv Res 18, 910.

[38] Wattmo C, Wallin AK, Londos E, Minthon L (2010) Risk factors for nursing home placement in Alzheimers disease: A longitudinal study of cognition, ADL, service utilization, and cholinesterase inhibitor treatment. Gerontologist 51, 17-27.

[39] Nielsen TR, Vogel A, Riepe MW, de Mendonça A, Rodriguez G, Nobili F, Gade A, Waldemar G (2011) Assessment of dementia in ethnic minority patients in Europe: A European Alzheimer's disease consortium survey. Int Psychogeriatr 23, 86-95.

[40] Fosbøl EL, Peterson ED, Holm E, Gislason GH, Zhang Y, Curtis LH, Køber L, Iwata I, Torp-Pedersen C, Setoguchi S (2012) Comparative cardiovascular safety of dementia medications: A cross-national study. J Am Geriatr Soc 60, 2283-2289.

[41] Ballard C, Lana MM, Theodoulou M, Douglas S, McShane R, Jacoby R, Kossakowski K, Yu LM, Juszczak E (2008) A randomised, blinded, placebo-controlled trial in dementia patients continuing or stopping neuroleptics (the DART-AD trial). PLoS Med 5, e76.

[42] Corbett A, Burns A, Ballard C (2014) Don't use antipsychotics routinely to treat agitation and aggression in people with dementia. BMJ 3, 349 .

[43] Essink-Bot M-L, Lamkaddem M, Jellema P, Smith Nielsen S, Stronks K (2012) Interpreting ethnic inequalities in healthcare consumption: A conceptual framework for research. Eur J Public Health 23, 922-926.

[44] Iliffe S, Manthorpe J (2004) The debate on ethnicity and dementia care: From category fallacy to person-centred care? Aging Mental Health 8, 283-292.

[45] Alzheimer Europe (2020) Intercultural dementia care - A guide to raise awareness amongst health and social care workers. Alzheimer Europe. 